\title{
Serum Osteoprotegerin and future risk of cancer and cancer-related mortality in the
}

$$
\text { general population - The Tromsø Study }
$$

Running title: Serum osteoprotegerin and future risk of cancer

The study is supported by independent grants from the University of Tromsø and the Northern Norway Regional Health Authority.

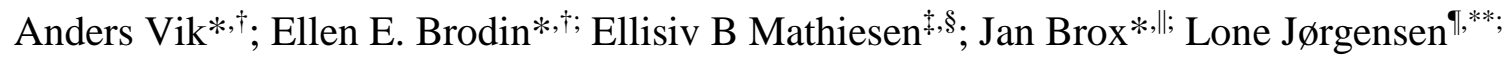
Inger Njølstad ${ }^{\dagger \dagger}$; Sigrid K. Brækkan*,†; John-Bjarne Hansen*,†

${ }^{*}$ Hematological Research Group (HERG), Department of Clinical Medicine, University of Tromsø, Norway.

${ }^{\dagger}$ Division of Internal Medicine, University Hospital of North Norway, Tromsø, Norway. ${ }^{\ddagger}$ Brain and Circulation Research Group, Department of Clinical Medicine, University of Tromsø, Norway.

${ }^{\S}$ Department of Neurology and clinical Neurophysiology, University Hospital of North Norway, Tromsø, Norway.

"Division of Laboraory Medicine, University Hospital of North Norway, Tromsø, Norway. `Department of Health and Care Sciences, University of Tromsø, Norway.

${ }^{* *}$ Department of Clinical Therapeutic Services, University hospital of North Norway, Tromsø, Norway.

${ }^{\dagger \dagger}$ Department of Community Medicine, University of Tromsø, Tromsø, Norway. Correspondence to: Anders Vik, Hematological Research Group (HERG), Department of Clinical Medicine, University of Tromsø, N-9037 Tromsø, Norway. Telephone: +47776 26305 Fax: +47 776 26831, e-mail: anders.vik@uit.no

The authors disclose no potential conflicts of interest

Word count: 3529. Number of tables and figures: 5+3 supplementary tables. 1 figure. 


\section{Abstract}

The purpose was to investigate the association between serum osteoprotegerin (OPG) and risk of incident cancer and cancer mortality in a general population. OPG was measured in serum collected from 6279 subjects without prior cancer recruited from a general population. Incident cancer and cancer-related mortality were registered from inclusion in 1994-95 until end of follow-up December 31, 2008. Cox regression models were used to estimate crude and adjusted (for age, sex and other confounders) hazard ratios and 95\% confidence intervals (HR; 95\% CI). There were 948 incident cancers and 387 deaths in the cohort during 71902 person-years of follow up (median 13.5 years). Subjects with serum OPG in the upper tertile had 79\% higher risk of incident gastrointestinal cancer than those in the lowest tertile (HR 1.79, 95\% CI 1.19-2.67). In women < 60 years, serum OPG (per SD $0.81 \mathrm{ng} / \mathrm{ml}$ ) was associated with reduced risk of incident cancer (all cancers merged) (0.73; 0.57-0.94) and breast cancer $(0.51 ; 0.31-0.83)$ after adjustment. Subjects in the upper tertile of OPG had higher risk of cancer-related mortality $(1.63 ; 1.16-2.28)$, particularly mortality from cancer in the gastrointestinal system $(2.28 ; 1.21-4.28)$ compared to those in the lowest OPG tertile. No significant association was detected between OPG and risk of death from cancer in the respiratory system or death from prostatic cancer. Our findings from a large population based cohort study suggest that serum OPG was associated with increased risk of incident gastrointestinal cancer, inversely associated with breast cancer, and predicts cancer-related mortality. 


\section{Background}

Osteoprotegerin (OPG) is a member of the tumor necrosis factor (TNF) receptor superfamily which exerts its function as a decoy receptor for receptor activator of nuclear factor kappa $\beta$ ligand (RANKL) and TNF-related apoptosis-inducing ligand (TRAIL) $(1,2)$. OPG inhibits ligation of RANKL and TRAIL to their cognate receptors, and thereby takes part in regulation of cell survival (2), bone homeostasis (3) and modulation of the immune system (4).

Experimental studies support the concept that OPG alone and its modulation of RANKL and TRAIL may affect risk of cancer. OPG synthesized by monocytes within tumors may promote survival of several tumor cell types (5-7), and in vitro studies indicate that OPG may act as a survival factor for tumor cells in both solid tumors $(5,8)$ and hematological malignancies (7), and induce angiogenesis $(9,10)$. TRAIL induces apoptosis in a variety of cells, and tumor cells are more sensitive than non-malignant cells $(9,11,12)$, whereas RANKL is involved in development and metastasis of breast cancer (13-15).

Recently, we reported that serum levels of OPG were associated with mortality of ischemic heart disease, stroke, and non-vascular causes (16). The latter finding may indicate that OPG is also a marker for other diseases as well as cardiovascular disease in the general population. Our observation that increasing serum OPG predicted mortality of non-vascular causes along with experimental evidence for a role of OPG-TRAIL-RANKL in carcinogenesis, elicited the hypothesis that serum levels of OPG may be positively associated with future risk of cancer and cancer-related mortality. To address this question, we investigated the association between serum OPG and future incident cancer and cancer-related mortality in a large population based cohort study during 14 years of follow-up. 


\section{Methods}

\section{Study population}

Participants were recruited from the fourth survey of the Tromsø Study conducted in 1994-95, a single-centre prospective, population-based study, with repeated health surveys of inhabitants in Tromsø, Norway. All inhabitants aged 55-74 years and 5-10\% samples in other 5-year age groups (25-54 years and 75-85 years) were invited to take part, and 78\% ( $\mathrm{n}=6899)$ of invited subjects attended. Subjects with cancer prior to baseline $(n=356)$ or incident cancer within 12 months after baseline $(n=72)$ were excluded to ensure that the presence of cancer or occult cancer would not affect levels of serum OPG. Further, subjects were excluded due to lack of consent to contribute to research $(n=57)$, not officially registered as inhabitants of the municipality of Tromsø ( $n=12)$, lack of serum samples for OPG measurement $(n=77)$, and development of malignant skin tumors during the follow up period ( $\mathrm{n}=46)$. Thus, 6279 subjects were included in our cohort study. Informed written consent was obtained from all participants, and the study was approved by the regional committee for research ethics. Incident cases of cancer and deaths from cancer among the participants were recorded from the date of enrolment through the end of follow-up, December $31^{\text {st }}, 2008$ and December $31^{\text {st }}$, 2007, respectively.

\section{Medical History, Blood Collection and Measurements}

Information on study participants was obtained by a self-administrated questionnaire, anthropometric measurements, and measurements of non-fasting blood samples (17). In brief, blood samples were collected from an antecubital vein and serum prepared by centrifugation after one hour respite at room temperature. OPG concentrations were analysed in freshlythawed serum aliquots stored at $-70^{\circ} \mathrm{C}$ for 12 years by an ELISA assay (R\&D Systems, 
Abingdon, UK) with mouse anti-human OPG as capture antibody. Biotinylated goat antihuman OPG and streptavidin horseradish peroxydase were used for detection. The OPG assay was performed according to the instructions by the manufacturer. The intra- and interassay coefficients of variation in our laboratory were $6.5 \%$ and $9.3 \%$, respectively. Between-assays variations in OPG were adjusted for by use of an internal standard. All samples were analyzed in duplicate and the mean value is used in this report. Serum lipids (total and HDL cholesterol, and triglycerides), haemoglobin A1c (HbA1c), high sensitivity C-reactive protein (hs-CRP) and creatinine were assessed as previously described (17).

\section{End point Assessment}

The national 11-digit identification number allowed linkage to the national registry of cancer and local diagnosis registries. Information on incident cancer during follow-up, e.g. date of cancer diagnosis and location of malignancy (ICD-7 codes 140-205), excluding nonmelanoma skin cancer (ICD-7 191.0-191.9), was obtained from the Cancer Registry of Norway (CRN). In a recent evaluation of the data quality, completeness of reporting was estimated to 98.8\%, whereas organ specific morphology had 94\% accuracy (18). Further, linkage to the National Causes of Death Registry at Statistics Norway and information from the death certificates was used to collect relevant information of the event. The Norwegian Registry of Vital Statistics provided information on emigration and death.

\section{Statistical Methods}

Continuous variables were presented as means (95\% confidence interval, or standard deviation (SD)), and categorical data as number or percentage. Multivariable linear or logistic regression models were used for sex and age adjustment, and to test for linear trends across tertiles of OPG for continuous and binary data, respectively. For each participant, person years of follow-up were calculated from the date of blood sampling in 1994-95, until the date 
of an event, the date the participant moved from the municipality of Tromsø, died, or until the end of the study period. Cox-proportional hazard regression models were used to estimate hazard ratios (HR), with 95\% confidence intervals (CI) for incident cancer, subtypes of cancer and cancer-related mortality in the entire population and in analyses stratified for sex and age categories ( $<60$ and $\geq 60$ years). OPG was treated as both categorized (tertiles) and continuous variable in the analyses. Crude analyses, analyses adjusted for age and sex (model 1), and multivariable analyses with variables shown to be associated with OPG (model 2: smoking, BMI, systolic blood pressure, HDL cholesterol, hsCRP, and self-reported diabetes mellitus/ HbA1c>6.1\%; model 3: model $2+$ history of ischemic stroke or myocardial infarction) were carried out. Possible two way interactions between sex, or age, with OPG was assessed by including cross product terms to the proportional hazards models. The proportional hazard assumption was verified by evaluating the parallelism between the curves of the log-log survivor function for tertiles of OPG. Subjects with incomplete data for the assessed covariates were excluded from the multivariable models. The statistical analyses were performed using SPSS software for Windows, version 18.0 (SPSS, Inc., Chicago, IL). Two sided $P$ values $<0.05$ were considered statistically significant. 


\section{Results}

The study population consisted of $50.5 \%(n=3174)$ women aged $61 \pm 10$ years with mean serum OPG concentration of $3.40 \pm 1.06 \mathrm{ng} / \mathrm{mL}$, and $49.5 \%(\mathrm{n}=3105)$ men aged $60 \pm 10$ years with mean serum OPG concentration of $3.21 \pm 1.17 \mathrm{ng} / \mathrm{mL}$ (P for sex difference $<0.0001$ ). Characteristics of participants at baseline (1994-95) across tertiles of serum OPG are shown in table 1. Age, blood pressure, HDL cholesterol, HbA1c, hsCRP, the proportion of women and subjects with diabetes mellitus increased, whereas body mass index (BMI) and triglycerides decreased significantly across tertiles of serum OPG.

There were 948 incident cases of cancer during a total of 71902 person-years of follow-up (median 13.5 years). The overall crude cancer incidence rate per 1000 person-years was 13.2 (95\% CI 12.4-14.1) in the total population, 9.9 (95\% CI 8.9-11.0) in women, and 16.8 (95\% CI 15.5-18.2) in men. The organ distribution of the most common cancers across tertiles of serum OPG is shown in table 2. The overall relative risk of incident cancer was 1.06 (95\% CI 0.99-1.15) per $1 \mathrm{SD}(1.12 \mathrm{ng} / \mathrm{mL})$ increase in serum OPG in age- and sex-adjusted analysis and 1.02 (0.94-1.12) in the multivariable adjusted model (model 3) (Table 3). Accordingly, there was no apparent change in risk estimates across tertiles of serum OPG ( $p$ for trend $=0.70$ ). Similar findings occurred in analyses stratified for sex.

Stratification for sex and age $(<60 \mathrm{yrs}$ and $\geq 60 \mathrm{yrs})$ revealed an inverse relation between serum OPG and incident cancer in women below 60 yrs of age (appendix table 1). The relative risk of cancer was 0.73 (95\% CI 0.57-0.94) per 1 SD $(0.81 \mathrm{ng} / \mathrm{ml})$ increase in serum OPG, whereas women in the upper tertile of serum OPG $(\geq 3.08 \mathrm{ng} / \mathrm{ml})$ had a relative risk of $0.44(0.26-0.75)$ of cancer compared to women in the lowest tertile $(\leq 2.42 \mathrm{ng} / \mathrm{ml})$ in the multivariable adjusted model (appendix table 1). In men below 60 years of age (appendix table 1 the relative risk of cancer was 1.10 (95\% CI 0.90-1.34) per 1 SD (0.99 ng/ml) increase 
in serum OPG, whereas men in the upper tertile of serum OPG ( $\geq 2.87 \mathrm{ng} / \mathrm{ml})$ had a relative risk of $1.48(0.95-2.31)$ of cancer compared to men in the lowest tertile $(\leq 2.33 \mathrm{ng} / \mathrm{ml})$ in the adjusted model (appendix table 1). No significant associations between serum OPG and risk of incident cancer were found in men and women above 60 years of age.

To investigate whether serum OPG was associated with particular types of cancer, we performed stratified analyses for the most common types of cancer (table 4). The relative risk of gastrointestinal cancers increased by $15 \%$ per 1 SD (1.12 ng/ml) increase in serum OPG, whereas those in the upper tertile of serum OPG $(\geq 3.55 \mathrm{ng} / \mathrm{ml})$ had $79 \%$ higher relative risk of cancer compared to subjects in the lowest tertile $(\leq 2.78 \mathrm{ng} / \mathrm{ml})$ in the adjusted model. The risk estimates were independent of age (appendix table 2). The cumulative incidence rates for gastrointestinal cancer progressively diverged across tertiles of serum OPG over time. Probability curves of survival free of gastrointestinal cancer are shown in figure 1, panel A. Colorectal cancer comprised 59\% $(n=164)$ of the gastrointestinal cancers. Subjects in the upper tertile of OPG had 49\% higher relative risk of colorectal cancer compared to subjects in the lowest tertile (p for trend 0.087). However, the strongest association between OPG and gastrointestinal cancer was found for esophagus $(n=16)$, stomach $(n=40)$ and pancreatic $(n=39)$ cancer merged. The relative risk increased by 37\% per 1 SD increase in serum OPG and was 2.55 fold higher in tertile 3 compared to tertile 1 (95\% CI; 1.23-5.30).

An inverse association between serum OPG and risk of breast cancer was observed (women only) (Table 4 and appendix table 2). The relative risk of breast cancer decreased by 26\% per $1 \mathrm{SD}(1.06 \mathrm{ng} / \mathrm{ml})$ increase in serum OPG, whereas women in the upper tertile of serum OPG ( $\geq 3.68 \mathrm{ng} / \mathrm{ml}$ ) had $45 \%$ lower relative risk of breast cancer compared to women in the lowest tertile $(<2.90 \mathrm{ng} / \mathrm{ml})$ in the adjusted model. The cumulative incidence rates for breast cancer progressively diverged across tertiles of serum OPG over time. Probability curves of survival 
free of breast cancer are shown in figure 1, panel B. In analyses stratified by age (appendix table 2), the protective effect of serum OPG on breast cancer applied to women below 60 years of age. The relative risk of breast cancer was 0.51 (95\% CI 0.31-0.83) per 1 SD (0.81 $\mathrm{ng} / \mathrm{ml})$ increase in serum OPG, and women in the upper tertile of serum OPG $(\geq 3.08 \mathrm{ng} / \mathrm{ml})$ had $76 \%$ reduced relative risk $(0.24$ (95\% CI $0.10-0.61))$ of breast cancer compared to women in the lowest tertile $(<2.42 \mathrm{ng} / \mathrm{ml})$ in this age group.

There were 387 subjects who died from cancer during follow up (median 12.6 years). The crude incidence rates of cancer-related mortality were 5.48 (95\% CI 4.96-6.05) in the total population, 3.86 (95\% CI 3.27- 4.55) in women and 7.22 (95\% CI 6.37- 8.18) in men per 1000 person-years, respectively. Serum OPG was associated with risk of cancer-related mortality irrespective of whether OPG was treated as a continuous or categorized variable (tertiles) (table 5). The overall relative risk of cancer-related mortality increased by 25\% per $1 \mathrm{SD}(1.12 \mathrm{ng} / \mathrm{mL})$ increase in serum OPG, and subjects in the upper tertile of serum OPG had $63 \%$ higher relative risk of dying from cancer than those within the lowest tertile of serum OPG. The cumulative incidence rates for cancer-related mortality progressively diverged across tertiles of serum OPG throughout the observation period. Probability curves of survival are shown in figure 1, panel C. The association between serum OPG and cancerrelated mortality was mostly driven by the relation to mortality from gastrointestinal cancers. The risk of dying from cancer in the gastrointestinal tract was 2.3 fold higher for subjects in the upper tertile of OPG compared to subjects in the lower tertile (p for trend=0.012). In women, no significant associations between OPG and death of all cancers merged were observed (appendix table 3). OPG tended to be associated with death of gastrointestinal cancer in women (increased 39\% per 1 SD OPG (95\% CI; 1.06-1.83) and almost 2 fold higher relative risk in tertile 3 versus tertile 1 of OPG). In contrast, in men a significant association between OPG and risk of dying of all cancers merged was observed, but no significant 
association between OPG and risk of dying of gastrointestinal cancer was found (appendix table 3). No significant association was observed between OPG levels and death from cancer in the respiratory system (table 5). Death from prostatic cancer occurred for 1 in the lower tertile of OPG, 15 in the middle tertile and 20 men in the upper tertile. However, no significant association was found (data not shown). Seven women died from breast cancer during the study period. 


\section{Discussion}

The present population based cohort study showed for the first time that serum OPG was associated with cancer-related mortality in general. Subgroup analyses revealed that this association in women was mostly driven by the relation to mortality from gastrointestinal cancers. However, serum OPG was not associated with risk of incident cancer in general, but displayed differential associations to gastrointestinal and breast cancer (women only). The relative risk of incident gastrointestinal cancer increased by 15\% per SD increase in serum OPG, and subjects in the upper tertile of serum OPG had 79\% higher relative risk than those in the lowest tertile. Subgroup analyses showed significant associations between OPG and oesophagus, stomach and pancreas cancer merged with 2.5 fold increased HR for subjects in the upper tertile compared to tertile 1 . Serum OPG was inversely associated with risk of breast cancer, particularly in women $<60$ years. No significant associations were observed between OPG and incident respiratory cancer, urinary tract cancer, prostatic cancer or genital cancer (women).

Although the potential impact of the OPG-RANKL-TRAIL axis on risk of cancer and cancerrelated mortality has been comprehensively studied in experimental models (5-15), few data are available from observational and interventional studies in humans. Previously, we observed an association between serum OPG and mortality from non-vascular causes in the general population (HR 1.31, 95\% CI 1.22-1.41) (16). Similar risk estimates, although not statistical significant, for mortality from non-vascular events were reported from the Bruneck cohort study including 915 subjects from the general population (19). The lack of significant associations in the Bruneck study between serum OPG and incident cancer and cancer related mortality may be due to low power caused by low number of events ( $n=146$ and $n=81$, respectively) (20). 
Our main finding that serum OPG was associated with risk of incident gastrointestinal cancers and mortality related to gastrointestinal cancers is supported by experimental and clinical studies. Increased expression of OPG has been reported in human gastric carcinoma (21) and colon cancer cell lines (22), as well as in patients with colorectal cancers and pancreatic cancers compared to healthy controls $(23,24)$. Addition of exogenous OPG to colorectal cancer cells caused resistance to TRAIL (23). Moreover, OPG mRNA expression was significantly higher in tumors with metastasis than without metastasis in both patients with gastric carcinoma (21) and colorectal cancer (25), and high expression of OPG was associated with decreased survival $(21,25)$ and increased risk of recurrence (25). Thus, our findings suggest that high serum OPG is an early biomarker of gastrointestinal cancers (more than a year ahead of diagnosis) and in women predicts mortality related to the disease.

Even though serum OPG was associated with higher risk of gastrointestinal cancers in women as well as in men, younger women $(<60$ years) showed an inverse relation between serum OPG and risk of incident total cancer. The inverse relation with total cancers in young women was mainly driven by incident breast cancer and cancer in the female genital organs. Experimental studies in breast cancer cells showed expression of OPG and inhibition of TRAIL induced apoptosis, suggesting that OPG may play a role in tumor cell survival (8). The expression of OPG in a large selection of breast tumors was also inversely correlated with tumor grade (8). Synthetic progesterone derivatives, such as medroxyprogesterone acetate (MPA), induced RANKL in mammary-gland epithelial cells in mice (13). Subsequent deletion of RANK from the mammary epithelium decreased incidence and delayed onset of MPA-driven mammary cancer (13). Moreover, selective inhibition of RANKL reduced preneoplasias as well as hormone- and carcinogen induced mammary epithelial proliferation 
(14). RANKL has also been shown to stimulate pulmonary metastasis of RANK(+) human breast cancer cells (15). Recently, it was shown in a mouse model for estrogen receptorpositive breast cancer that RANKL inhibition by OPG-Fc treatment blocked osteoclast activity and prevented tumor-induced osteolysis. In a combination experiment, OPG-Fc and tamoxifen resulted in a more efficient inhibition of tumor growth than either single agent alone (26). Thus, it has been proposed that inhibition of RANKL-RANK signalling might be used in conjunction with elimination of primary breast tumors to prevent recurrent metastatic disease (15). Furthermore, microarray analysis of 295 primary breast cancer patients revealed that high OPG mRNA levels correlated with differentiated tumors and longer overall- and disease-free survival (27).

In agreement with the animal models $(13-15,26)$, our population based study indicate that the cytokine network OPG is part of might also be important in development of tumors, and suggest that the inverse relation between serum OPG and risk of breast cancer, might be explained by reduced binding of RANKL to RANK in subjects with higher concentration of OPG.

Among patients with prostatic cancer serum OPG levels were significantly elevated in patients with bone metastasis and predicted prostatic cancer related death (28). In our population based cohorts study, 178 subjects developed prostatic cancer during follow up. We observed no significant association between OPG and incident prostatic cancer. The relatively high number of events makes a type II error unlikely.

In the present study, 167 subjects were diagnosed with cancer in the respiratory system and 132 of them died from their cancer during the observation period. No associations were observed between serum OPG and risk of cancer and cancer-related mortality in the 
respiratory system. In human non-small cell lung cancer (NSCLC) tissue samples, significantly stronger immunostaining for OPG, RANKL and RANK were observed in bone metastases than in tumor cells at the primary site (29). Furthermore, the RANKL:OPG ratios were significantly higher in bone metastases compared with primary NSCLC tissue samples (29).

The differential impact of OPG in various types of cancer might partly be explained by different significance of TRAIL induced apoptosis. For instance, many colorectal cancers show resistance to TRAIL partly due to expression of antagonistic decoy receptors (30). The effect of OPG varies according to expression rates, spatial distribution of receptors, decoys and ligands (31). An epidemiological study will never be able to disentangle the important balance between local and systemic actions of cytokines. However, it is intriguing that serum concentrations of OPG, in the present population based study, years before cancer development are associated with gastrointestinal cancer and breast cancer. The introduction of a monoclonal antibody (denosumab) to RANKL for the treatment of osteoporosis and reduction of skeletal events in subjects with breast cancer and prostatic cancer treated with hormones, underline the importance of further research.

Major advantages of the present study includes the population-based design, high number of participants, long term follow-up and the completeness of end-point registration. Some limitations also merit consideration. Despite the high number of participants, the number of subjects in some cancer groups was relatively low. Potential confounders and assumptions for statistical models were carefully checked, however potential residual confounding cannot be completely ruled out. It is uncertain whether our findings in a European Caucasian population are generalizable to other ethnical groups. OPG was measured only at one time point and 
potentially our risk estimates could be somewhat underestimated due to regression dilution. Non- fasting blood samples and non-standardized time points for sampling could introduce bias. In young normolipemic males the serum concentration of OPG decreased modestly during the day (32). In the postprandial state after a lipid rich meal we have found a similar modest decrease in serum OPG. Thus, it is unlikely that non-fasting blood samples and various time points for sampling during the day have introduced severe bias. Serum samples were kept frozen for 12 years at $-70^{\circ} \mathrm{C}$ without any freezing-thawing cycles before measurement of OPG and this may have influenced the measurements. However, previous studies have reported long-term stability of OPG measurements in serum samples stored at $70^{\circ} \mathrm{C}(33)$

In conclusion, serum OPG was associated with incident cancer and death from cancer in the gastrointestinal system, and inversely associated with incident breast cancer in young women. Our findings support that OPG is a biomarker for development of certain cancers and that OPG predicts cancer-related mortality. 


\section{Legend to figure}

\section{Figure 1}

Probability of survival free of: A; gastrointestinal cancer, B; breast cancer and C; cancer related mortality stratified by OPG tertiles. Adjusted for age, sex, smoking, BMI, systolic blood pressure, HDL cholesterol, CRP, diabetes mellitus or HbA1c $>6.1 \%$, ischemic stroke and/or myocardial infarction before baseline. P for trend 0.004, 0.08 and 0.007, respectively. 


\section{References}

1. Yasuda H, Shima N, Nakagawa N, et al. Osteoclast differentiation factor is a ligand for osteoprotegerin/osteoclastogenesis-inhibitory factor and is identical to TRANCE/RANKL. PNAS. 1998;95(7):3597-602.

2. Emery JG, McDonnell P, Burke MB, et al. Osteoprotegerin Is a Receptor for the Cytotoxic Ligand TRAIL. J. Biol. Chem. 1998;273(23):14363-7.

3. Simonet WS, Lacey DL, Dunstan CR, et al. Osteoprotegerin: A Novel Secreted Protein Involved in the Regulation of Bone Density. Cell. 1997;89(2):309-19.

4. Reid P, Holen I. Pathophysiological roles of osteoprotegerin (OPG). Eur J Cell Biol. 2009;88(1):1-17.

5. Holen I, Croucher PI, Hamdy FC, Eaton CL. Osteoprotegerin (OPG) is a survival factor for human prostate cancer cells. Cancer Res. 2002;62(6):1619-23.

6. Neville-Webbe HL, Cross NA, Eaton CL, et al. Osteoprotegerin (OPG) produced by bone marrow stromal cells protects breast cancer cells from TRAIL-induced apoptosis. Breast Cancer Res Treat. 2004;86(3):269-79.

7. Shipman CM, Croucher PI. Osteoprotegerin is a soluble decoy receptor for tumor necrosis factor-related apoptosis-inducing ligand/Apo2 ligand and can function as a paracrine survival factor for human myeloma cells. Cancer Res. 2003;63(5):912-6.

8. Holen I, Cross SS, Neville-Webbe HL, et al. Osteoprotegerin (OPG) expression by breast cancer cells in vitro and breast tumours in vivo--a role in tumour cell survival? Breast Cancer Res Treat. 2005;92(3):207-15.

9. Cross SS, Yang Z, Brown NJ, et al. Osteoprotegerin (OPG)--a potential new role in the regulation of endothelial cell phenotype and tumour angiogenesis? Int J Cancer. 2006;118(8):1901-8. 
10. Benslimane-Ahmim Z, Heymann D, Dizier B, et al. Osteoprotegerin, a new actor in vasculogenesis, stimulates endothelial colony-forming cells properties. J Thromb Haemost. 2011;9(4):834-43.

11. Wiley SR, Schooley K, Smolak PJ, et al. Identification and characterization of a new member of the TNF family that induces apoptosis. Immunity. 1995;3(6):673-82.

12. Pitti RM, Marsters SA, Ruppert S, Donahue CJ, Moore A, Ashkenazi A. Induction of apoptosis by Apo-2 ligand, a new member of the tumor necrosis factor cytokine family. J Biol Chem. 1996;271(22):12687-90.

13. Schramek D, Leibbrandt A, Sigl V, et al. Osteoclast differentiation factor RANKL controls development of progestin-driven mammary cancer. Nature. 2010;468(7320):98-102.

14. Gonzalez-Suarez E, Jacob AP, Jones J, et al. RANK ligand mediates progestininduced mammary epithelial proliferation and carcinogenesis. Nature. 2010;468(7320):103-7. 15. Tan W, Zhang W, Strasner A, et al. Tumour-infiltrating regulatory T cells stimulate mammary cancer metastasis through RANKL-RANK signalling. Nature. 2011;470(7335):548-53.

16. Vik A, Mathiesen EB, Brox J, et al. Serum osteoprotegerin is a predictor for incident cardiovascular disease and mortality in a general population: the Tromso Study. J Thromb Haemost. 2011;9(4):638-44.

17. Vik A, Mathiesen EB, Johnsen SH, et al. Serum osteoprotegerin, sRANKL and carotid plaque formation and growth in a general population - The Tromso Study. Journal of Thrombosis and Haemostasis. 2010;8(5):898-905.

18. Larsen IK, Småstuen M, Johannesen TB, et al. Data quality at the Cancer Registry of Norway: An overview of comparability, completeness, validity and timeliness. European Journal of Cancer. 2009;45(7):1218-31. 
19. Kiechl S, Schett G, Wenning G, et al. Osteoprotegerin is a risk factor for progressive atherosclerosis and cardiovascular disease. Circulation. 2004;109(18):2175-80.

20. Kiechl S, Willeit J, Schett G, et al. Denosumab, Osteoporosis, and Prevention of Fractures. The New England journal of medicine. 2009;361(22):2188-91.

21. Ito R, Nakayama H, Yoshida K, et al. Expression of osteoprotegerin correlates with aggressiveness and poor prognosis of gastric carcinoma. Virchows Arch. 2003;443(2):146-51. 22. Pettersen I, Bakkelund W, Smedsrød B, Sveinbjørnsson B. Osteoprotegerin is Expressed in Colon Carcinoma Cells. Anticancer Research. 2005;25(6B):3809-16.

23. De Toni EN, Thieme SE, Herbst A, et al. OPG is regulated by beta-catenin and mediates resistance to TRAIL-induced apoptosis in colon cancer. Clinical cancer research : an official journal of the American Association for Cancer Research. 2008;14(15):4713-8.

24. Lipton A, Ali SM, Leitzel K, et al. Serum osteoprotegerin levels in healthy controls and cancer patients. Clinical cancer research : an official journal of the American Association for Cancer Research. 2002;8(7):2306-10.

25. Tsukamoto S, Ishikawa T, Iida S, et al. Clinical Significance of Osteoprotegerin Expression in Human Colorectal Cancer. Clinical Cancer Research. 2011;17(8):2444-50.

26. Canon J, Bryant R, Roudier M, Branstetter DG, Dougall WC. RANKL inhibition combined with tamoxifen treatment increases anti-tumor efficacy and prevents tumor-induced bone destruction in an estrogen receptor-positive breast cancer bone metastasis model. Breast Cancer Res Treat. 2012;135(3):771-80.

27. Santini D, Schiavon G, Vincenzi B, et al. Receptor activator of NF-kB (RANK) expression in primary tumors associates with bone metastasis occurrence in breast cancer patients. PLoS One. 2011;6(4):e19234. 
28. Kamiya N, Suzuki H, Endo T, et al. Significance of serum osteoprotegerin and receptor activator of nuclear factor $\kappa \mathrm{B}$ ligand in Japanese prostate cancer patients with bone metastasis. International Journal of Clinical Oncology. 2011:1-7.

29. Peng X, Guo W, Ren T, et al. Differential expression of the RANKL/RANK/OPG system is associated with bone metastasis in human non-small cell lung cancer. PLoS One. 2013;8(3):e58361.

30. Van Geelen CM, de Vries EG, de Jong S. Lessons from TRAIL-resistance mechanisms in colorectal cancer cells: paving the road to patient-tailored therapy. Drug resistance updates : reviews and commentaries in antimicrobial and anticancer chemotherapy. 2004;7(6):345-58.

31. Ryser MD, Qu Y, Komarova SV. Osteoprotegerin in bone metastases: mathematical solution to the puzzle. PLoS computational biology. 2012;8(10):e1002703.

32. Vik A, Brodin E, Sveinbjornsson B, Hansen JB. Heparin induces mobilization of osteoprotegerin into the circulation. Thromb Haemost. 2007;98(1):148-54. doi:07070148 [pii] 33. Kiechl S, Schett G, Schwaiger J, et al. Soluble receptor activator of nuclear factorkappa B ligand and risk for cardiovascular disease. Circulation. 2007;116(4):385-91. 